\title{
Tsallis and Kaniadakis Entropic Measures in Polytropic, Logarithmic and Exponential Functions
}

\section{Amelia Carolina Sparavigna ${ }^{1}$}

\author{
${ }^{1}$ Department of Applied Science and Technology, Politecnico di Torino, Torino, Italy
}

\begin{abstract}
Among nonextensive statistical approaches, those proposed by Constantino Tsallis and Giorgio Kaniadakis had been involved in the study of several physical phenomena. Here, we will discuss the case of the polytropic solutions of self-gravitating fluid spheres used in astrophysics as approximate stellar models. We will see how, in this problem, Tsallis and Kaniadakis entropic measures are related. After, following the same approach, we will find the links between generalized logarithms and exponential functions of the abovementioned entropic measures.
\end{abstract}

Keywords: Entropy, Generalized Entropies

\section{Introduction}

The nonextensive statistical mechanics is a research on foundations of statistical mechanics, which is based on the generalization of the well-known Boltzmann--Gibbs theory. This statistical mechanics, which is formulated with different approaches, enables the study of systems with long-range interactions, long-term memories or multi-fractal structures [1]. The Tsallis and Kaniadakis approaches are two of the nonextensive generalizations of statistical mechanics, which had been involved in the discussion of several phenomena [1,2]. Among the possible examples, here we consider one from astrophysics. It is related to polytropes, the polytropic solutions of the Lane-Emden equation. This is an equation which gives the pressure as a function of density [3]. These solutions are modelling selfgravitating fluid spheres that are called "polytropes" too, which are objects used as approximation to more realistic stellar models [4]. The solution of the LaneEmden equation, a dimensionless form of Poisson's equation for the gravitational potential, depends on a parameter known as the polytropic index $n$. It is usually written as $P=K \rho^{(n+1) / n}$, where $P$ is pressure, $\rho$ is density and $K$ a constant. If stellar structure is approximated with a polytrope having a given index, two scaling parameters are needed to express the structure in physical units [5]. The two parameters that we can use are a constant related to the entropy and the stellar mass. Since Boltzmann distribution yields unphysical results, a generalized entropy, the Tsallis entropy, was used in [6] instead of Boltzmann entropy. The use of Kaniadakis entropy had been recently proposed too, in [7]. Here we will see that these two entropic measures are related in polytropic solutions, and that the result given in [7] can be easily obtained from [6]. After, following the same approach used for polytropes, we will see how the generalized logarithms and exponential functions, used in these two nonextensive methods, are linked. Of course, this procedure can be easily repeated for all the generalized functions, based on the abovementioned measures.

\section{The entropies}

Well-known is the entropy proposed by Claude Shannon in 1948 [8]. He defined the entropy $H$ of a discrete random variable $X$, as the expected value of the information content: $H(X)=-\sum_{i} p_{i} \log _{b} p_{i}$

. The probability of i-event is $p_{i}$ and $\mathrm{b}$ is the base of the used logarithm. However, several entropies exist which are generalizing Shannon entropy. Among them we have Tsallis and Kaniadakis entropies [9,10], which are defined, with a corresponding choice of measurement units equal to 1, as follow:

(1) Tsallis :

$$
\mathrm{T}=\mathrm{T}_{\mathrm{q}}=\frac{1}{\mathrm{q}-1}\left(1-\sum_{\mathrm{i}} \mathrm{p}_{\mathrm{i}}^{\mathrm{q}}\right)
$$


(2) Kaniadakis ( $\kappa$ - entropy) :

$$
\mathrm{K}_{\kappa}=-\sum_{\mathrm{i}} \frac{\mathrm{p}_{\mathrm{i}}^{1+\kappa}-\mathrm{p}_{\mathrm{i}}^{1-\kappa}}{2 \kappa}
$$

In (1) and (2) we have the entropic indices q and $\kappa$. For its generalized additivity, the Kaniadakis entropy requires another function, defined as follow: $\mathfrak{I}=\sum_{\mathrm{i}}\left(\mathrm{p}_{\mathrm{i}}^{1+\kappa}+\mathrm{p}_{\mathrm{i}}^{1-\kappa}\right) / 2$. A detailed discussion of the generalized additivity of Tsallis and $\kappa$-entropy is given in [11].

Tsallis and Kaniadakis entropies are linked:

$$
\mathrm{K}_{\kappa}=\frac{\mathrm{T}_{1+\kappa}+\mathrm{T}_{1-\kappa}}{2}
$$

Where:

$$
\begin{aligned}
& \mathrm{T}(\mathrm{q}=1+\kappa)=-\frac{1}{\kappa} \sum_{\mathrm{i}} \mathrm{p}_{\mathrm{i}}^{1+\kappa}+\frac{1}{\kappa} \\
& \mathrm{T}(\mathrm{q}=1-\kappa)=\frac{1}{\kappa} \sum_{\mathrm{i}} \mathrm{p}_{\mathrm{i}}^{1-\kappa}-\frac{1}{\kappa}
\end{aligned}
$$

Eq.(3) is a simpler form of an expression given in $[12,13]$. However, besides this relation, because of the generalized additivity possessed by the Kaniadakis entropy, we need also another relation:

$$
\mathfrak{I}_{\kappa}=\frac{\kappa}{2}\left(-\mathrm{T}_{1+\kappa}+\mathrm{T}_{1-\kappa}+\frac{2}{\kappa}\right)
$$

In (3) and (4), we have Kaniadakis functions expressed by Tsallis entropy. As shown in [14], we can also write $\mathrm{T}$ expressed by means of Kaniadakis functions:

$$
\mathrm{K}_{\kappa}+\frac{1}{\kappa} \mathfrak{J}_{\kappa}=\mathrm{T}_{1-\kappa}+\frac{1}{\kappa}
$$

Let us have: $\kappa=1-q$. From (5) we have immediately the relation between Tsallis entropy and Kaniadakis entropic measures:

(6) $\mathrm{T}_{\mathrm{q}}=\mathrm{K}_{1-\mathrm{q}}+\frac{\mathfrak{I}_{1-\mathrm{q}}-1}{(1-\mathrm{q})}$

\section{With polytropes}

The relation (6) between Tsallis and Kaniadakis entropies can be useful in several problems. Here we consider its use in polytropes. In the previous equations, we have $p_{i}$ denoting the probability distribution. In Ref.6, it is used letter $\mathrm{f}$ for probability. From now on, we will use this notation. In [6], the measure from Tsallis entropy is:

(7) $\mathrm{C}_{\mathrm{q}}(\mathrm{f})=\frac{\mathrm{f}\left(1-\mathrm{f}^{\mathrm{q}-1}\right)}{\mathrm{q}-1}$

We can write Eq.7 in the following manner:

(8) $\mathrm{C}_{\mathrm{q}}(\mathrm{f})=\frac{\mathrm{f}-\mathrm{f}^{\mathrm{q}}}{\mathrm{q}-1}=\frac{1}{2(\mathrm{q}-1)}\left(\mathrm{f}^{2-\mathrm{q}}-\mathrm{f}^{\mathrm{q}}\right)-\frac{1}{2(\mathrm{q}-1)}\left(\mathrm{f}^{2-\mathrm{q}}+\mathrm{f}^{\mathrm{q}}\right)+\frac{\mathrm{f}}{\mathrm{q}-1}$

Of course, (7) and (8) are the same equation. Therefore, Kaniadakis measures are linked to Tsallis measure by:

$$
\text { (9) } \mathrm{t}_{\mathrm{q}}(\mathrm{f})=\mathrm{C}_{\mathrm{q}}(\mathrm{f})=\frac{\left(\mathrm{f}-\mathrm{f}^{\mathrm{q}}\right)}{\mathrm{q}-1}=\mathrm{k}_{1-\mathrm{q}}-\frac{\mathrm{g}_{1-\mathrm{q}}}{\mathrm{q}-1}+\frac{\mathrm{f}}{\mathrm{q}-1}
$$

Where:

$$
\mathrm{T}_{\mathrm{q}}=\sum_{\mathrm{f}} \mathrm{t}_{\mathrm{q}}, \mathrm{K}_{1-\mathrm{q}}=\sum_{\mathrm{f}} \mathrm{k}_{1-\mathrm{q}}, \mathfrak{J}_{1-\mathrm{q}}=\sum_{\mathrm{f}} \mathrm{g}_{1-\mathrm{q}}, 1=\sum_{\mathrm{f}} \mathrm{f}
$$

From [6], a relation exists between polytrope index and entropic Tsallis index:

$$
\text { (10) } \mathrm{n}=\frac{3}{2}+\frac{1}{\mathrm{q}-1}
$$

As a special case, for $q \rightarrow 1$, we find the isothermal situation. To have Eq.6, as shown in [14], we need $\kappa=1-\mathrm{q}$ or $\kappa=\mathrm{q}-1$. Then, from (10), considering that we have for the Kaniadakis index $-1<\kappa<1$, we find:

(11) $\mathrm{n}=\frac{3}{2}+\frac{1}{\kappa}$

In fact, (11) is the relation that we find in [7]. Using 
then the relation between Tsallis and Kaniadakis measures we can easily finds results concerning several applications. Polytropes are an example of such possible approach.

\section{Logarithms}

Following the example of polytropes, we can investigate the relation between generalized logarithms. In the framework of Tsallis approach, the logarithm is defined as:

(12) $\ln _{\mathrm{q}}^{\mathrm{T}}(\mathrm{f})=\frac{\mathrm{f}^{1-\mathrm{q}}-1}{1-\mathrm{q}}$
In the Kaniadakis approach:

(13) $\ln _{\kappa}^{K}(f)=\frac{f^{\kappa}-f^{-\kappa}}{2 \kappa}$

Let us write (13), with $\kappa=1-\mathrm{q}$ :

(14) $\ln _{1-\mathrm{q}}^{\mathrm{K}}(\mathrm{f})=\frac{\mathrm{f}^{1-\mathrm{q}}-\mathrm{f}^{\mathrm{q}-1}}{2(1-\mathrm{q})}$

As we did for polytropes:

(15) $\quad \ln _{\mathrm{q}}^{\mathrm{T}}(\mathrm{f})=\frac{1-\mathrm{f}^{1-\mathrm{q}}}{\mathrm{q}-1}=\frac{1}{2(\mathrm{q}-1)}\left(\mathrm{f}^{\mathrm{q}-1}-\mathrm{f}^{1-\mathrm{q}}\right)-\frac{1}{2(\mathrm{q}-1)}\left(\mathrm{f}^{\mathrm{q}-1}+\mathrm{f}^{1-\mathrm{q}}\right)+\frac{1}{\mathrm{q}-1}$

$$
=\ln _{1-q}^{K}(f)+\frac{f^{1-q}+f^{q-1}}{2(1-q)}+\frac{1}{q-1}=\ln _{1-q}^{K}(f)+\frac{1}{f} \frac{f^{2-q}+f^{q}}{2(1-q)}+\frac{1}{q-1}
$$

Here we find again, in (15), the measures found in (8). Then, we can write, as we did in (9):

$$
\ln _{q}^{T}(f)=\ln _{1-q}^{K}(f)+\frac{1}{f} \frac{g_{1-q}}{(1-q)}-\frac{1}{1-q}
$$

In (16), we have the relation between Tsallis and Kaniadakis generalized logarithms.

\section{Exponentials}

In the framework of Tsallis approach, the exponential is defined as:

$$
e_{q}^{T}(f)=[1+(1-q) f]^{1 /(1-q)}
$$

We have that: $e_{q}^{T}\left(\ln _{\mathrm{q}}^{\mathrm{T}}(\mathrm{f})\right)=\mathrm{f}$. So:

$$
\begin{aligned}
e_{\mathrm{q}}^{\mathrm{T}}\left(\ln _{\mathrm{q}}^{\mathrm{T}}(\mathrm{f})\right) & =\left[1+(1-\mathrm{q})\left(\ln _{1-\mathrm{q}}^{\mathrm{K}}(\mathrm{f})+\frac{1}{\mathrm{f}} \frac{\mathrm{g}_{1-\mathrm{q}}}{(1-\mathrm{q})}-\frac{1}{1-\mathrm{q}}\right)\right]^{1 /(1-\mathrm{q})} \\
& =\left[(1-\mathrm{q}) \ln _{1-\mathrm{q}}^{\mathrm{K}}(\mathrm{f})+\frac{\mathrm{g}_{1-\mathrm{q}}}{\mathrm{f}}\right]^{1 /(1-\mathrm{q})}=\mathrm{f}
\end{aligned}
$$

Therefore, we have also the relation (considering $\kappa=1-\mathrm{q}$ ):

$$
\kappa \ln _{\kappa}^{\mathrm{K}}(\mathrm{f})=-\frac{g_{\kappa}}{\mathrm{f}}+\mathrm{f}^{\kappa}
$$

In (18) we have an expression of the generalized logarithm, containing the Kaniadakis measure $\mathrm{g}_{\kappa}$, where $\mathfrak{I}_{\kappa}=\sum_{\mathrm{f}} \mathrm{g}_{\kappa} \cdot(18)$ is in agreement with (16).

Let us consider the exponential from Kaniadakis measure:

(19) $\mathrm{e}_{\kappa}^{\mathrm{K}}(\mathrm{f})=\left[\sqrt{1+\kappa^{2} \mathrm{f}^{2}}+\kappa \mathrm{f}\right]^{1 / \kappa}$ 
We have also $\mathrm{e}_{\kappa}^{\mathrm{K}}\left(\ln { }_{\kappa}^{\mathrm{K}}(\mathrm{f})\right)=\mathrm{f}$, and then:

$$
e_{\kappa}^{K}(\ln \underset{\kappa}{K}(f))=\left[\sqrt{1+\kappa^{2}\left(\frac{f^{\kappa}-f^{-\kappa}}{2 \kappa}\right)^{2}}+\kappa\left(\frac{f^{\kappa}-f^{-\kappa}}{2 \kappa}\right)\right]^{1 / \kappa}=\left[\frac{1}{f} g_{\kappa}+\kappa \frac{1}{f} k_{\kappa}\right]^{1 / \kappa}=f
$$

Let us remember that we have defined: $\mathrm{K}_{\kappa}=\sum_{\mathrm{f}} \mathrm{k}_{\kappa}, \mathfrak{I}_{\kappa}=\sum_{\mathrm{f}} \mathrm{g}_{\kappa}$.

However:

$$
\text { (21) }\left[\frac{1}{\mathrm{f}} \mathrm{g}_{\kappa}+\kappa \frac{1}{\mathrm{f}} \mathrm{k}_{\kappa}\right]=\mathrm{f}^{\kappa} \rightarrow \mathrm{g}_{\kappa}+\kappa \mathrm{k}_{\kappa}=\mathrm{f}^{\kappa+1}
$$

Then, we can find the following relation between the Tsallis exponential and the Kaniadakis measures:

$$
\text { (22) } e_{q}^{T}(f)=e_{q}^{T}\left(e_{1-q}^{K} \ln _{1-q}^{K}(f)\right)=\left[1+(1-q)\left(\frac{g_{1-q}}{f}+(1-q) \frac{k_{1-q}}{f}\right)^{1 /(1-q)}\right]^{1 /(1-q)}
$$

This is a link between Tsallis exponential function and Kaniadakis measures. In fact:

$$
e_{q}^{T}(f)=e_{q}^{T}\left(e_{1-q}^{K} \ln _{1-q}^{K}(f)\right)=\left[1+(1-q)\left(f^{(1-q)}\right)^{1 /(1-q)}\right]^{1 /(1-q)}
$$

In the previous discussion, following the same approach used for polytropes, we have seen that generalized logarithms and exponential functions, used by Tsallis and Kaniadakis entropic measures, are linked. Of course, the same approach can be used for all the generalized functions that we can obtain in the framework of the abovementioned measures.

\section{References}

1) Sumiyoshi, A. and Yuko O., Editors (2001). Nonextensive Statistical Mechanics and Its Applications, Springer Science \& Business Media, ISBN: 978-3-540-41208-3 (Print) 978-3540-40919-9 (Online)

2) Kaniadakis, G. (2013). Theoretical Foundations and Mathematical Formalism of the Power-law Tailed Statistical Distributions. Entropy 15(10):3983-4010. DOI: 10.3390/e15103983

3) Horedt, G.P. ( 2004). Polytropes. Applications in Astrophysics and Related Fields, Kluwer. ISBN 1-40202350-2

4) Chandrasekhar, S. (1958). An Introduction to the Study of Stellar Structure, Dover.

5) Knapp, J. (2015). Polytropes, retrieved 9 November 2015 from http://www.astro.princeton.edu/ gk/A403/polytrop.pdf

6) Plastino, A.R.; Plastino, A. (1993). Stellar Polytropes and Tsallis Entropy. Physics Letters A 174(5):384-386. DOI: 10.1016/0375-9601(93)90195-6
7) Bento, E.P.; Silva, J.R.P.; Silva, R. (2013). Non-Gaussian Statistics, Maxwellian Derivation and Stellar Polytropes. Physica A: Statistical Mechanics and its Applications 392.4 (2013): 666-672. DOI: 10.1016/j.physa.2012.10.022

8) Shannon, C.E. (1948). A Mathematical Theory of Communication. Bell System Technical Journal 2(3):379423. DOI: $10.1002 / j .1538-7305.1948 . t b 01338 . x$

9) Tsallis, C. (1960). Possible Generalization of BoltzmannGibbs Statistics, Journal of Statistical Physics, 1988, 52: 479-487. DOI:10.1007/BF01016429

10) Kaniadakis, G.(2002). Statistical Mechanics in the Context of Special Relativity, Phys. Rev. E, 2002, 66, 056125. DOI: 10.1103/physreve.66.056125

11) Sparavigna, A.C. (2015). On the Generalized Additivity of Kaniadakis Entropy, Int. J. Sci. 4(2):44-48. DOI: 10.18483/ijSci.627

12) Kaniadakis, G. (2001). Non-Linear Kinetics Underlying Generalized Statistics, Physica A 296(3-4):405-425. DOI: 10.1016/s0378-4371(01)00184-4

13) Santos, P.; Silva R.; Alcaniz J.S.; Anselmo, D.H.A.L. (2011). Generalized Quantum Entropies, Physics Letters A 375:3119-3123. DOI: 10.1016/j.physleta.2011.07.001

14) Sparavigna, A.C. (2015). Relations Between Tsallis and Kaniadakis Entropic Measures and Rigorous Discussion of Conditional Kaniadakis Entropy, Int. J. Sci. 4(10):47-50. DOI: $10.18483 /$ ijsci.866 